\title{
A Phase 2 Randomized Trial of Asleep versus Awake Subthalamic Nucleus Deep Brain Stimulation for Parkinson's Disease
}

\author{
Julien Engelhardt ${ }^{a, b, c}$ François Caire ${ }^{k, I} \quad$ Nathalie Damon-Perrière ${ }^{b, c, d}$ \\ Dominique Guehl $^{b, c, d}$ Olivier Branchard ${ }^{a}$ Nicolas Auzou $^{e, n} \quad$ François Tison $^{b, c, e}$ \\ Wassilios G. Meissnerb, c, e Elsa Krim ${ }^{f}$ Stéphanie Bannierg ${ }^{\text {Antoine Bénard }}{ }^{\text {h }}$ \\ Rémi Sittah $^{\text {h Denys Fontaine }}{ }^{\mathrm{i}, j} \quad$ Xavier Hoarau $^{m} \quad$ Pierre Burbaud $^{\text {b, }}$, d \\ Emmanuel Cuny ${ }^{a, b, c}$
}

${ }^{a} \mathrm{CHU}$ de Bordeaux, Service de Neurochirurgie B, Bordeaux, France; 'b Institut des Maladies Neurodégénératives, Université de Bordeaux, UMR 5293, Bordeaux, France; 'CCNRS, Institut des Maladies Neurodégénératives, UMR 5293, Bordeaux, France; ${ }^{\mathrm{C}} \mathrm{CHU}$ de Bordeaux, Service d'explorations Fonctionnelles du Système Nerveux, Bordeaux, France; ${ }^{e} \mathrm{CHU}$ de Bordeaux, Service de Neurologie, Bordeaux, France; ${ }^{\mathrm{f}} \mathrm{CH}$ de Pau, Service de Neurologie, Pau, France; ${ }^{9} \mathrm{CH}$ de Bayonne, Service de Neurologie, Bayonne, France; ${ }^{\mathrm{h}} \mathrm{CHU}$ Bordeaux, Pôle de Santé Publique, Clinical Epidemiology Unit (USMR), Bordeaux, France; 'CHU de Nice, Service de Neurochirurgie, Nice, France; 'Université Côte d'Azur, Nice, France; 'Université de Limoges, CNRS, XLIM, UMR7252, Limoges, France; 'CHU de Limoges, Service de Neurochirurgie, Limoges, France; ${ }^{m}$ Polyclinique de Navarre, Service de Neurochirurgie, Pau, France; ${ }^{\text {LLaboratoire de }}$ Psychologie, Université de Bordeaux, Bordeaux, France

\section{Keywords}

Deep brain stimulation · Subthalamic nucleus · Parkinson's disease - Awake surgery · Asleep surgery · Microelectrode recordings

\begin{abstract}
Objective: Asleep deep brain stimulation (DBS) for Parkinson's disease (PD) is being performed more frequently; however, motor outcomes and safety of asleep DBS have never been assessed in a prospective randomized trial. Methods: We conducted a prospective, randomized, noncomparative trial to assess the motor outcomes of asleep DBS. Leads were implanted in the subthalamic nucleus (STN) according to probabilistic stereotactic coordinates with a surgical robot under $\mathrm{O}$-arm ${ }^{\odot}$ imaging guidance under either general anes-
\end{abstract}

thesia without microelectrode recordings (MER) (20 patients, asleep group) or local anesthesia with MER and clinical testing (9 patients, awake group). Results: The mean motor improvement rates on the Unified Parkinson's Disease Rating Scale Part III (UPDRS-3) between OFF and ON stimulation without medication were 52.3\% (95\% Cl: 45.4-59.2\%) in the asleep group and $47.0 \%$ (95\% Cl: $23.8-70.2 \%)$ in the awake group, 6 months after surgery. Except for a subcutaneous hematoma, we did not observe any complications related to the surgery. Three patients (33\%) in the awake group and 8 in the asleep group (40\%) had at least one side effect potentially linked with neurostimulation. Conclusions: Owing to its randomized design, our study supports the hypothesis that motor outcomes after asleep STN-DBS in PD may be noninferior to the standard awake procedure.

(c) 2020 S. Karger AG, Base

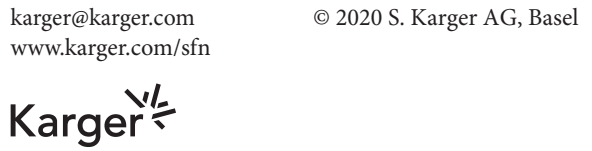

Dr. Julien Engelhardt

Service de Neurochirurgie B - Hôpital Pellegrin

Place Amélie Raba-Léon

Bordeaux Cédex 33076 (France)

Julien.engelhardt@chu-bordeaux.fr 


\section{Introduction}

Subthalamic nucleus (STN) deep brain stimulation (DBS) has evolved over the past decades as a mainstream therapy for advanced Parkinson's disease (PD) with $>50 \%$ of improvement in the Unified Parkinson's Disease Rating Scale Part III (UPDRS III) scale in prospective randomized trials [1-9] and up to $60 \%$ in a retrospective study [10]. The standard established DBS procedure is based on awake surgery with intraoperative microelectrode recording (MER) associated with clinical testing to ensure good positioning of the lead [11]. However, this procedure is sometimes perceived as uncomfortable for patients who are unable to tolerate awake surgery with extensive time off their medication. This may limit the surgical option for a significant proportion of potential candidates for surgery. Awake surgery carries a potential risk of hemorrhage due to the use of several microelectrode tracks [12] and is more costly than asleep DBS surgery due to the length of the procedure and availability of suitably trained staff [13]. Indeed, asleep STN-DBS without MER is being performed with increasing frequency [14-18].

Analysis of the literature including retrospective, prospective nonrandomized studies, meta-analyses, and literature reviews [19-26] suggests that there is no significant difference in motor outcomes between asleep and awake STN-DBS. Asleep surgery may lead to lower overall surgical complication rates (i.e., intracerebral hemorrhage and infection), but awake procedure may lead to fewer stimulation-induced side effects [23]. On the other hand, it has been recently demonstrated that despite significant advances in STN visualization on imaging [27-29], intraoperative electrophysiology may still be useful for optimal control of targets [30]. Indeed, no definitive conclusion can be reached due to the lack of a prospective randomized clinical trial comparing the efficacy and safety of these 2 techniques.

Therefore, we performed a prospective randomized trial, in which patients eligible for STN-DBS surgery for $\mathrm{PD}$ were randomly assigned to undergo surgery according to the asleep (general anesthesia [GA] without MER) or awake (local anesthesia [LA] with MER and clinical testing) procedure. Motor outcomes were assessed at 6 months postoperatively according to the UPDRS-3 scale.

\section{Methods}

\section{Study Design}

The PARKEO trial was a prospective phase 2 open-label singlecenter randomized noncomparative trial with 2 parallel arms (awake and asleep surgery). Currently, this design is more often performed than single-arm phase 2 trials $[31,32]$. The PARKEO trial was conducted at the Institute for Neurodegenerative Disorders and the Department of Neurosurgery of the Bordeaux University Hospital, France.

\section{Patients}

Patients with a diagnosis of idiopathic PD at the motor fluctuation stage despite optimal medical treatment and L-DOPA sensitivity were eligible for enrollment. L-DOPA sensitivity was defined as motor improvement above $50 \%$ on the UPDRS- 3 scale after a dose of $150 \%$ of the usual early morning treatment. Indication for STN-DBS was approved by the local multidisciplinary movement disorders committee including neurologists, neurosurgeons, and neuropsychologists. Patients between 18 and 70 years of age could be included. Exclusion criteria were significant cognitive decline defined by a score of $<130$ on the Mattis Dementia Rating Scale (MDRS), mood disorders defined by a score of $>20$ on the Beck Depression Inventory (BDI), significant cortical atrophy visualized on brain MRI, contraindication to anesthesia, unstoppable anticoagulant or platelet antiaggregant treatment, and lack of contraceptive treatment for women of childbearing age.

\section{Randomization and Masking}

Patients were randomly assigned to either the asleep or the awake group in a 2:1 ratio in favor of the asleep group. An unbalanced randomization ratio was chosen because of the extensive knowledge on the awake DBS procedure. We wanted to gather more information on the innovative asleep procedure and improve the precision of the confidence intervals. Randomization was performed centrally via a web-based system. Twenty patients were allocated to the asleep group, and 10 patients were allocated to the awake group. Clinical motor evaluation was blindly performed at 6 months after surgery by 2 neurologists specializing in PD (N.D.P. and D.G.) not involved in the intraoperative electrophysiological procedure (P.B.). One neuropsychologist specializing in PD (N.A.) performed MDRS and BDI assessments.

\section{Procedures}

Surgical Procedure

For all patients, a Leksell frame $\left(\right.$ Elekta $\left.^{\complement}\right)$ was positioned (under LA in the awake group and GA in the asleep group), and then a T1 MRI $\left(\mathrm{TR}=15 \mathrm{~ms}, \mathrm{TE}=7 \mathrm{~ms}\right.$, matrix $512 \times 512$, flip angle $30^{\circ}$, bandwidth $15 \mathrm{kHz}, \mathrm{NEX}=1$, and slice sickness $1.5 \mathrm{~mm}$ ) at 1.5 Tesla (Siemens ${ }^{\mathrm{TM}}$ Achieva) was performed. Coordinates of the STN target were calculated in the anterior commissure-posterior commissure (AC-PC) stereotactic space using a probabilistic clinical-based STN target developed in our center [33].

For the asleep group, the Leksell frame was fixed to the surgical robot Neuromate ${ }^{\mathrm{TM}}$. After a $5-\mathrm{cm}$ robot-guided incision, a burr hole of $2.5 \mathrm{~mm}$ in diameter was made. Then, the guide of the Medtronic $^{\odot} 3389$ electrode was inserted into the clinical-based target using the robot and under radiographic control with the $\mathrm{O}$ arm $\left(\right.$ Medtronic $^{\circledR}$ ) scanner. The 3389 Medtronic $^{\circledR}$ electrode of 40 $\mathrm{cm}$ in length was inserted into the guide tube to the planned target (radiographic control). After removal of the guide tube, the electrode was fixed by a silk suture passed through the bone before tunneling under the scalp. The electrode position was checked during the procedure by front and side $2 \mathrm{D} \mathrm{O}$-arm ${ }^{\odot}$ radiographic control and at the end of the procedure with a $3 \mathrm{D}$ intraoperative $\mathrm{O}$-arm ${ }^{\odot} \mathrm{CT}$ scan fused with the MRI scan performed at the begin- 
ning of the procedure. Immediately after implantation of the 2 electrodes, the second part of the surgery involved setting up the generator in the left paraumbilical region. Then, the electrode and its extensions were tunneled under the skin to the stimulator and connected.

For the awake group, the Leksell frame was fixed to the surgical robot Neuromate ${ }^{\mathrm{TM}}$. After a $5-\mathrm{cm}$ robot-guided incision, a burr hole of $14 \mathrm{~mm}$ in diameter was made and the 5 microelectrode guides were inserted using the robot under 2D front and side radiographic control O-arm ${ }^{\odot}$ (Medtronic $^{\mathrm{TM}}$ ). Electrophysiological recordings began $10 \mathrm{~mm}$ above the planned target. Neuronal activity was stored at different levels, and somesthetic receptive fields of STN neurons were sought. Depending on the electrophysiology results, stepped stimulations using 3-5 electrodes were performed. They made it possible to quantify (1) the clinical efficacy on rigidity and tremor when present and (2) possible side effects of stimulation (maximal intensity used, $7 \mu \mathrm{A}$ ). The final target was chosen on the basis of the best clinical effects without side effects. A 3D intraoperative $\mathrm{O}$-arm $\left(\right.$ Medtronic $^{\odot}$ ) scanner fused with the MRI scan performed at the beginning of the procedure was used to check the positioning of the electrophysiological target as regard to the planned target and to calculate the frame-based coordinates of the electrophysiological target. The 3389 Medtronic $\left.^{(}\right)$electrode of $40 \mathrm{~cm}$ in length was inserted into the guide tube to the defined target (radiographic control). It was fixed using an osteosynthesis 6-hole plate $\left(\right.$ Biomet $^{\complement}$ ref 01-7050) before tunneling under the scalp. The final electrode position was then checked using a second $3 \mathrm{D} O$-arm ${ }^{\odot}$ scanner acquisition fused with the MRI scan performed at the beginning of the procedure. The same procedure was used for the contralateral side. The second surgery was performed in a different session ( 5 days later) under GA and consisted in placing the stimulator in the left paraumbilical region and connecting it with the extensions and electrodes.

Stereotactic Coordinates for STN Targeting

The stereotactic coordinates of the target were expressed as a function of 3 landmarks well visible on a T1 MRI at 1.5 tesla (for further details on the definition of these coordinates, please refer to online suppl. material; for all online suppl. material, see www. karger.com/doi/10.1159/000511424 and references [33, 34]):

- $x_{\text {target }}=0.44 x_{\text {lateral edge of the third ventricle }}+10.71 \mathrm{~mm}$, at the midcommissural point

$-y_{\text {target }}=0.69 y_{\text {mammillothalamic tract }}+1.62 \mathrm{~mm}, 4 \mathrm{~mm}$ below the AC-PC line

- $z_{\text {target }}=0.72 \times$ thalamus height $-16 \mathrm{~mm}$

\section{Other Procedures}

DBS device programming and monitoring as well as any medical treatment, including another surgery procedure, deemed necessary for the patient were authorized and performed according to standard clinical practice. The study protocol had no predefined stereotactic error threshold for lead repositioning. Reintervention for lead repositioning was indicated based on clinical and stereotactic (error) considerations in our multidisciplinary staff.

\section{Outcomes}

The primary endpoint was the efficacy of the surgical procedure on motor symptoms assessed by the change in the UPDRS-3 scores between OFF and ON stimulation evaluations at 6 months after surgery without any medical treatment (OFF medication).
The secondary endpoints were assessed 6 months after the surgery and included the following: (1) the failure rate defined by the proportion of patients with an improvement in the UPDRS-3 score between OFF and ON stimulation of $<35 \%$ without any medical treatment, (2) the reduction in the levodopa equivalent daily doses (LEDD) from the baseline (a 100-mg daily dose of standard levodopa was equivalent to the following doses of other medications: $133 \mathrm{mg}$ of controlled-release levodopa; $75 \mathrm{mg}$ of levodopa plus entacapone; $1 \mathrm{mg}$ of pergolide, pramipexole lisuride, or cabergoline; $5 \mathrm{mg}$ of ropinirole; $10 \mathrm{mg}$ of bromocriptine or apomorphine; and $20 \mathrm{mg}$ of dihydroergocryptine), (3) the improvement from baseline in quality of life measured by the Parkinson Disease Questionnaire 39 (PDQ-39), (4) the effects of the stimulation on cognition and mood (measured by the MDRS and BDI scales, respectively, and compared with the baseline score), and (5) side effects associated with the surgical procedure or neurostimulation. We also calculated the stereotactic error as the minimal distance " $d$ " between the predefined target before surgery and the axis of the DBS lead. The distance $d$ is given by the following formula, in ACPC referential:

$$
d=\|\overrightarrow{B A} \wedge \vec{u}\|,
$$

where $\vec{u}$ is the unitary director vector of the DBS lead and $\overrightarrow{B A}$ is the vector between $A$ (the predefined target) and $B$ (the center of the proximal contact of the lead).

\section{Statistical Analysis}

Sample size was based on feasibility, and an unbalanced allocation was planned to allow for a precision of $\pm 6.6 \%$ for the $95 \%$ CI of the primary outcome in the asleep group, assuming a standard deviation of $15 \%$. All analyses were carried out in accordance with the intention-to-treat principle, in which all randomized participants are analyzed according to their randomization group. Missing data for the primary endpoint were considered a failure (thus replaced by the worst observed value). As a phase 2 study, the purpose of the randomization was to reduce bias due to patient selection into either procedure group, and the sample size did not allow proving the equivalence between the 2 procedures. Therefore, according to latest guidelines for Statistical Reporting [35], the results are provided without $p$ value. Statistical analyses were performed using SAS software v9.4 (SAS Institute, Cary, NC, USA).

\section{Standard Protocol Approvals, Registrations, and Patient \\ Consents}

The trial protocol was approved by the regional scientific ethics committee (Comité de Protection des Personnes Sud-Ouest et Outre-Mer III) and was performed in accordance with the principles of the Declaration of Helsinki. This study is registered with ClinicalTrials.gov, No. NCT01817088. Written informed consent was obtained from all patients.

\section{Data Availability Policy}

The protocol of this study does not foresee that individual deidentified participant data will be shared (reference framework number 001 of the C.N.I.L. - the French Data Protection Authority); however, the study protocol (in French), the statistical analysis plan (in French), and the informed consent form (in French) could be shared after validation by the Scientific Council of this study. 


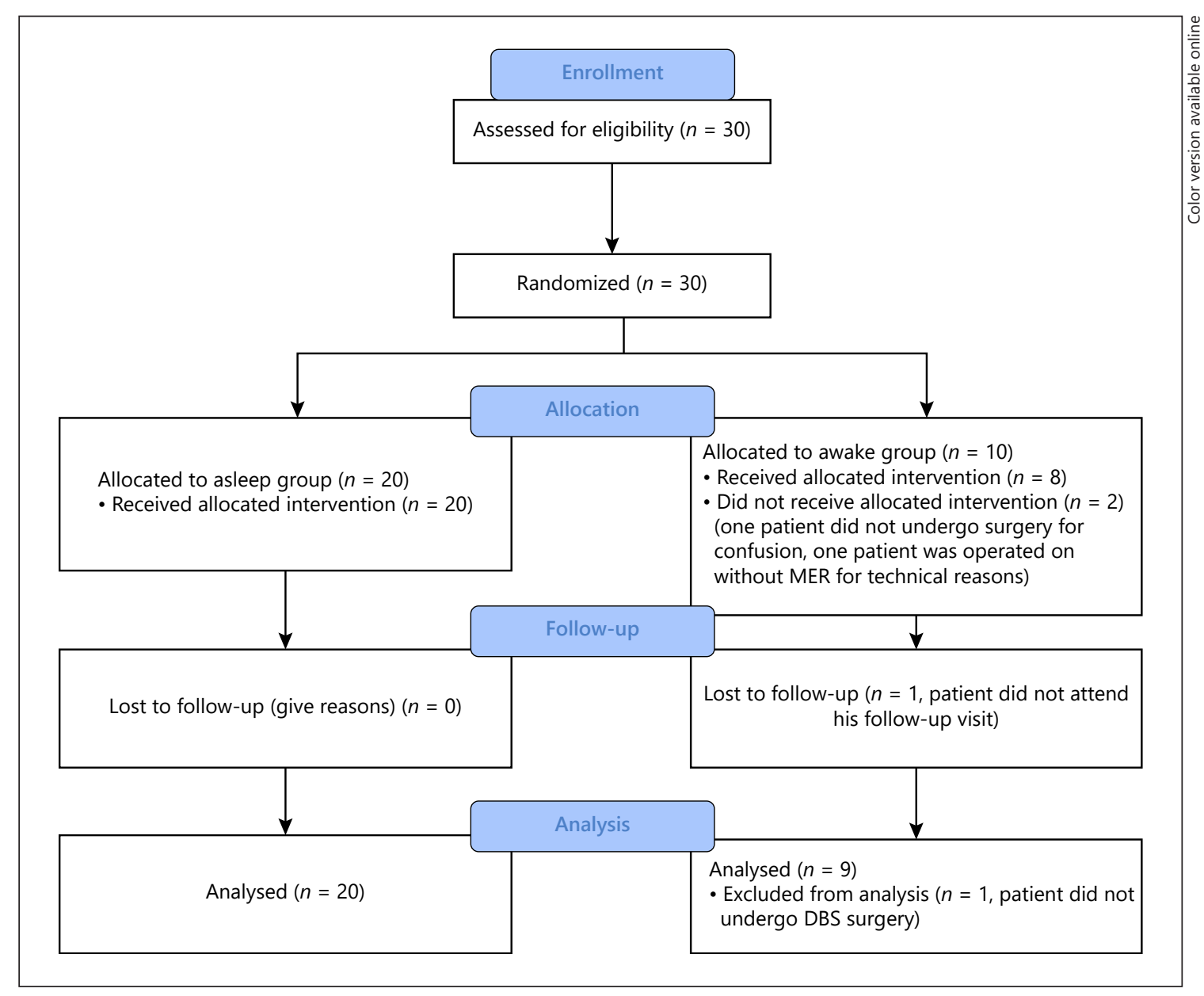

Fig. 1. CONSORT 2010 flow diagram. MER, microelectrode recordings; DBS, deep brain stimulation.

\section{Results}

\section{Characteristics of the Patients}

Thirty patients were enrolled in this trial between March 2013 and February 2016 (Fig. 1). Over the study period, 77 consecutive patients underwent a DBS procedure in our center. There were 10 DBS for dystonia, 17 for essential tremor, and 2 for obsessive compulsive disorder. Among the 48 patients with PD, 4 were implanted in the GPi and 6 were reoperated on for previous complications (infection and dysfunction). A total of 39 consecutive patients were theoretically eligible, but 9 were not included mainly because of exclusion criteria for microelectrode recordings:

- Atrophy and hypersignals on T2 FLAIR on brain MRI for 4 patients

Asleep versus Awake Subthalamic

Nucleus Deep Brain Stimulation
- Four patients whose condition was too severe without medication, who therefore could not have undergone the intervention under LA OFF medication

- Refusal to participate (1 patient)

Twenty patients were randomized to the asleep group and 10 to the awake group. One patient (awake group) did not undergo surgery due to preoperative confusion and was excluded from the study. One patient assigned to the awake group did not undergo MER for technical reasons and was implanted according to the "asleep" technique; he was, however, included in the awake group according to the intention-to-treat analysis. One patient randomized to the awake group was implanted on one side only and did not attend his follow-up visit at 6 months and was considered a failure in the intention-totreat analysis. The diagnoses of 2 patients were revised to multiple system atrophy disease during follow-up (1 pa- 
Table 1. Baseline patient characteristics of the 2 randomized groups

\begin{tabular}{lcc}
\hline Group & Asleep $(n=20)$ & Awake $(n=9)$ \\
\hline Sex ratio (M/F) & 2.33 & 2 \\
Age, years & $60(56-66)$ & $63(55-67)$ \\
UPDRS-3 OFF medication & $33.8(24.5-47.5)$ & $33.0(28.5-42.0)$ \\
UPDRS-3 ON medication & $10.5(4.3-16.5)$ & $8.0(7.0-11.0)$ \\
LEDD, mg/day & $1,362(929-1,583)$ & $837(795-1,197)$ \\
PDQ-39 & $58(43-70)$ & $61(44-81)$ \\
Beck Depression Index & $6(4-10)$ & $6(4-10)$ \\
Mattis Dementia Rating Scale & $142(139-143)$ & $135(135-141)$ \\
Duration of the disease, years & $12(9-14)$ & $10(9-11)$ \\
\hline
\end{tabular}

Data are expressed as median (interquartile range). UPDRS-3, Unified Parkinson's Disease Rating Scale Part III; LEDD, levodopa equivalent daily doses; PDQ-39, Parkinson Disease Questionnaire 39. tient from each group), but these patients were included in the analysis according to the intention-to-treat principle. Baseline population characteristics are described in Table 1.

\section{Motor Outcomes}

The motor improvement rate (mean [95\% CI]) between OFF and ON stimulation (OFF medication) at 6 months was $52.3 \%(45.4-59.2 \%)$ in the asleep group, $47.0 \%(23.8-70.2 \%)$ in the awake group, and $50.6 \%$ (42.9$58.4 \%)$ for all patients. The median differences (interquartile range) between UPDRS-3 scores between OFF and $\mathrm{ON}$ stimulation (OFF medication) at 6 months were 22.0 (16.0-31.8) and 15.5 (13.5-29.0), in the asleep group and in the awake group, respectively (Table 2 ).

In addition, the failure rates (percentage [exact binomial CI]) were $10 \%(1.2-31.7 \%)$ in the asleep group (2 patients) and $33.3 \%$ (7.5-70.1\%) in the awake group (3 patients). The decrease in LEDD (mean [95\% CI]) was $22.9 \%(8.9-36.9 \%)$ in the asleep group and $22.2 \%(-3.4$ to $47.9 \%$ ) in the awake group (Table 2).

\section{Effect on Quality of Life, Cognition, and Depression}

The decrease in PDQ-39 (mean [95\% CI]) was -18.8 $(-31.6$ to -6.0$)$ in the asleep group and $-9.6(-36.4$ to 17.2 ) in the awake group (Table 2). The MDRS scores of 2 patients worsened, falling below 130: 1 in the asleep group (127) and 1 in the awake group (129). The 6-month MDRS evaluation showed a median score (interquartile range) of 140.0 (138.0-143.0) in the asleep group and 135.5 (133.0-139.5) in the awake group versus 142 (139$143)$ and $135(135-141)$ at baseline, respectively. The 6-month BDI evaluation showed a median score (inter- quartile range) of 6.0 (3.5-11.0) in the asleep group and $5.0(1.5-12.5)$ in the awake group versus $6(4-10)$ and 6 (4-10) at baseline, respectively.

\section{Surgical Complications and Side Effects Related to Neurostimulation}

Except for a subcutaneous abdominal hematoma under the stimulator, which required a puncture of the collection (1 patient, asleep group), we did not observe any complication directly linked to the surgical procedure in this study. One patient was reoperated on because of postoperative freezing and walking disorders despite a significant decrease in dyskinesia, but without any benefit from the second intervention (asleep arm), and 1 patient for failure of implantation in one side due to a technical incident (awake arm). Eleven patients (38\%) presented with at least one side effect potentially associated with the neurostimulation: 3 patients in the awake group (33\%) and 8 patients in the asleep group (40\%).

The different side effects were as follows: worsening of akinesia in 2 patients (asleep group), dysarthria in 3 patients (asleep group), dyskinesia in 3 patients (asleep group), worsening of motor fluctuations in 1 patient (awake group), increased freezing periods in 1 patient (asleep group), orthostatic hypotension in 3 patients (asleep group) - with one isolated episode during immediate postoperative time in 1 patient, repeated episodes in 1 patient, and one case of severe hypotension immediately following the L-DOPA test - dysexecutive syndrome and apathy in 1 patient (awake group), suicidal ideation in 1 patient (awake group), hypersexuality in 1 patient (asleep group), and weight gain of $4 \mathrm{~kg}$ in the 3 months following surgery in 1 patient (asleep group). 
Table 2. Results on primary and secondary endpoints in the 2 randomized groups

\begin{tabular}{|c|c|c|}
\hline Group & Asleep $(n=20)$ & Awake $(n=9)$ \\
\hline Primary endpoint & Missing & Missing $=1^{a}$ \\
\hline UPDRS-3 OFF medication ratio ${ }^{\mathrm{b}}$ & $52.3(45.4 ; 59.2)$ & $47.0(23.8 ; 70.2)$ \\
\hline UPDRS-3 OFF-ON stimulation (OFF medication) & $22.0(16.0 ; 31.8)$ & $15.5(13.5 ; 29.0)$ \\
\hline UPDRS-3 OFF medication OFF stimulation & $43.8(37.5 ; 49.5)$ & $37.5(26.8 ; 43.0)$ \\
\hline UPDRS-3 ON medication OFF stimulation & $13.5(9.0 ; 22.5)$ & $17.0(11.3 ; 22.8)$ \\
\hline UPDRS-3 OFF medication ON stimulation & $21.8(16.0 ; 23.3)$ & $16.5(8.3 ; 26.5)$ \\
\hline UPDRS-3 ON medication ON stimulation & $10.5(8.5 ; 14.0)$ & $11.8(8.0 ; 15.8)$ \\
\hline \multicolumn{3}{|l|}{ Secondary endpoints } \\
\hline Failure rate, $n$ & 2 & 3 \\
\hline Failure rate, $\%^{c}$ & $10.0(1.2-31.7)$ & $33.3(7.5-70.1)$ \\
\hline$L E D D^{b}$ & Missing $=0$ & Missing $=1$ \\
\hline LEDD absolute reduction, mg/day & $341(145.7 ; 536.2)$ & $224.5(-40.4 ; 489.4)$ \\
\hline LEDD reduction ratio & $22.9(8.9 ; 36.9)$ & $22.2(-3.4 ; 47.9)$ \\
\hline \multicolumn{3}{|l|}{$P D Q-39$} \\
\hline \multirow[t]{2}{*}{ Baseline } & Missing $=2$ & Missing $=4$ \\
\hline & $58(43 ; 70)$ & $61(44 ; 81)$ \\
\hline \multirow[t]{2}{*}{6 months } & Missing $=1$ & Missing $=1$ \\
\hline & $37.0(23.0 ; 56.0)$ & $43.5(32.0 ; 59.5)$ \\
\hline Evolution $^{\mathrm{b}}$ & Missing $=3$ & Missing $=4$ \\
\hline Absolute difference (M6-M0) & $-18.8(-31.6 ;-6.0)$ & $-9.6(-36.4 ; 17.2)$ \\
\hline \multicolumn{3}{|l|}{$M D R S$} \\
\hline \multirow{2}{*}{ Baseline } & Missing $=0$ & Missing $=0$ \\
\hline & $142(139 ; 143)$ & $135(135 ; 141)$ \\
\hline \multirow[t]{2}{*}{6 months } & Missing $=5$ & Missing $=1$ \\
\hline & $140.0(138.0 ; 143.0)$ & $135.5(133.0 ; 139.5)$ \\
\hline Evolution $^{\mathrm{b}}$ & Missing $=5$ & Missing $=1$ \\
\hline Absolute difference (M6-M0) & $-2.1(-4.2 ; 0.1)$ & $-2.1(-4.6 ; 0.4)$ \\
\hline \multicolumn{3}{|l|}{$B D I$} \\
\hline \multirow[t]{2}{*}{ Baseline } & Missing $=1$ & Missing $=0$ \\
\hline & $6(4 ; 10)$ & $6(4 ; 10)$ \\
\hline \multirow[t]{2}{*}{6 months } & Missing $=4$ & Missing $=1$ \\
\hline & $6.0(3.5 ; 11.0)$ & $5.0(1.5 ; 12.5)$ \\
\hline Evolution $^{\mathrm{b}}$ & Missing $=5$ & Missing $=1$ \\
\hline Absolute difference (M6-M0) & $-1.0(-4.3 ; 2.3)$ & $0.4(-4.8 ; 5.5)$ \\
\hline \multicolumn{3}{|c|}{ 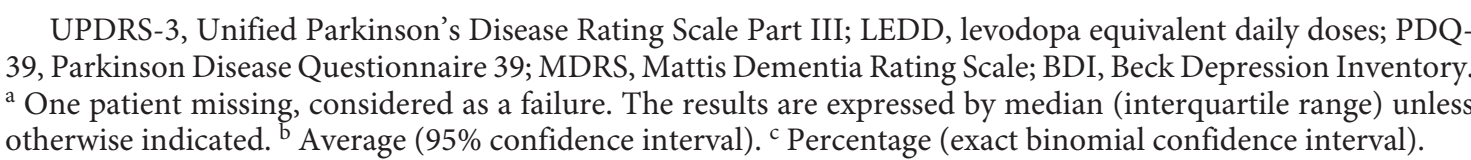 } \\
\hline
\end{tabular}

\section{Duration of Surgery, Radiation Doses,}

Pneumocephalus, and Stereotactic Error

In the asleep group, the duration of surgery was of $4 \mathrm{~h}$ $50 \mathrm{~min} \pm 37 \mathrm{~min}$ (lead implantation and stimulator im- plantation during the same surgery). In the awake group, duration was $6 \mathrm{~h} 58 \mathrm{~min} \pm 1 \mathrm{~h} 1 \mathrm{~min}$ for lead implantation (first surgery, under LA) plus $22 \pm 7$ min for stimulator implantation (second surgery, under GA). The mean 
dose of irradiation received by patients during surgery measured as the dose-length product (DLP) was $260.8 \pm$ $92.3 \mathrm{mGy} \mathrm{cm}$ in the asleep group and $547.7 \pm 175.9 \mathrm{mGy}$ $\mathrm{cm}$ in the awake group. Pneumocephalus was measured on the 3D O-arm preoperative scanner. A significant pneumocephalus was observed in 4 patients of the awake group (44\%) and in no patient of the asleep group (0\%), $p=0.005$ (exact Fisher test). The mean stereotactic error in the awake cohort and the asleep cohort was $1.08 \pm 0.52$ and $1.38 \pm 0.58 \mathrm{~mm}$, respectively $(p=0.08$, Student $t$ test $)$.

\section{Discussion}

The results of this prospective randomized noncomparative phase 2 clinical trial showed that STN-DBS surgery based on a targeting procedure using clinical patientbased stereotactic coordinates coupled with lead placement by a surgical robot and intraoperative $\mathrm{O}$-arm ${ }^{\odot}$ imaging guidance provides an improvement rate $(95 \% \mathrm{CI})$ between OFF and ON stimulation (OFF medication) at 6 months of 52.3\% (45.4-59.2\%) when leads are directly implanted to the target under GA without MER correction and clinical testing. Contrary to single-group phase 2 clinical trials or case series where the interpretation is made only in relation to historical comparators, we designed our study so as to interpret the results of the asleep group with respect to those of the awake group, with said groups presenting the same characteristics (due to randomization) and being recruited and followed in the same settings (parallel groups), which strengthened our design.

Our results are comparable with the improvement rates observed in the surgical groups of the randomized trials that compared STN-DBS with MER versus optimal medical management. Indeed, Deuschl et al. [2] found improvement rates between preoperative and 6-month postoperative OFF-medication $\mathrm{ON}$-stimulation evaluations to be $41 \%$ in the UPDRS-3 scale, $24 \%$ in the PDQ39, and 49\% in the LEDD; Schüpbach et al. [7] in 2007 found rates to be 59,24 , and $71 \%$, respectively, in a first study; and Weaver et al. [8] found improvement to be 28, 20 , and $23 \%$, respectively. At 12 months, improvement rates between preoperative and postoperative OFF-medication ON-stimulation evaluation were found to be $36 \%$ in the UPDRS-3 scale, 13\% in the PDQ-39, and 34\% in the LEDD, in the study by Williams et al. [9]. At 24 months, improvement rates were 38,26 , and $39 \%$, respectively, in a second study by Schüpbach et al. [6]. However, we observed a smaller reduction in LEDD treatment (22.9\%) in our study as compared to the $23-71 \%$ in previ- ous studies. This can be explained by the fact that, in our practice, we do not drastically decrease dopaminergic treatment doses in order to avoid potential side effects (such as apathy).

Our results are also in accordance with the other studies that compared the outcomes after asleep and awake STNDBS. The meta-analysis of Ho et al. [23] showed mean improvement rates in UPDRS-3 scores in the OFF-medication $\mathrm{ON}$-stimulation condition between pre- and postoperative assessments of $51.1 \pm 16.6 \%$ in the asleep group (510 patients) versus $46.7 \pm 27.4 \%$ in the awake group $(4,931 \mathrm{pa}$ tients). However, the authors found an increased proportion of side effects due to neurostimulation in the asleep group as measured by the UPDRS-IV OFF-medication evaluation and a higher rate of surgical complications in the awake group. Another meta-analysis of Liu et al. [24] which analyzed the outcomes of 967 patients under GA versus 556 under LA reported in 14 studies found that there was no significant difference between the GA and LA groups in motor function improvement, levodopa requirements, and adverse events (including adverse events related to stimulation, general neurological and surgical complications, and hardware-related complications).

In our study, we found that stimulation-related complications were slightly higher in the asleep group and that qualitatively there were also differences with motor complications which were higher. This could be the consequence of a suboptimal positioning of the lead or a random effect due to the small sample size of the study. In any case, no definitive conclusion can be drawn because stimulation-related complications were secondary outcome measures and the study was not powered to answer this question. However, consideration of the side effects remains of paramount importance when comparing outcomes between awake and asleep DBS. For instance, in our study, some side effects might have been avoided in the asleep group if preoperative clinical testing had been performed. During awake surgery, it is possible to look for side effects that will assist in targeting (internal capsule, diplopia, autonomic effects, dysarthria, and paresthesia). In general, the side effects which are found in the operating room at the position of the definitive lead will be retrieved postoperatively. For the following side effects, we can hypothesize that they could have been prevented by preoperative testing: worsening of akinesia in 2 patients, dysarthria in 3 patients, dyskinesia in 3 patients, and orthostatic hypotension in 3 patients, with one isolated episode during immediate postoperative time in 1 patient, repeated episodes in 1 patient, and one case of severe hypotension immediately following the L-DOPA 
test. For the other ones, these kinds of side effects can happen even with preoperative testing because it is not possible to look for freezing or hypersexuality or weight gain during the intervention: increased freezing periods in 1 patient, hypersexuality in 1 patient, and weight gain of $4 \mathrm{~kg}$ in the 3 months following surgery in 1 patient. Finally, maybe directional leads could reduce the incidence of side effects. Directional leads are designed to theoretically correct a suboptimal positioning of the DBS-leads in order to maximize the therapeutic window and thus decrease the incidence of stimulation-related side effects. The errors might be corrected in the sagittal and lateral planes only if the electrode is close enough the target. In some cases, this kind of lead may have been interesting. However, the clinical benefit of directional leads remains to be proven in prospective randomized trials.

However, the literature remains difficult to interpret and summarize $[22,36]$ and conclusions must be drawn with caution because comparisons are made between different centers with a huge heterogeneity between surgical techniques (i.e., direct image-based targeting with different imaging modalities (T2, T2*, SWI, FGATIR, etc.)/indirect targeting based on different atlases or statistical stereotactic coordinates, frame/frameless procedure, use of surgical robot or not, MER with intraoperative stimulation, intraoperative stimulation only, MER under GA, intraoperative imaging guidance by $\mathrm{O}-\mathrm{arm}^{\odot}$, CT scan, MRI, etc.), between clinical outcomes (motor outcomes according to the UPDRS-3 or LEDD, quality of life according to the PDQ-39, and only a few studies studying other important factors such as speech and cognition), and between lengths of follow-up. It is also important to note that retrospective studies comparing awake and asleep DBS have often compared asleep DBS using modern intraoperative imaging to awake DBS with MER without advanced intraoperative imaging (historical cohorts). However, in our study, the awake group (MER + clinical stimulation) was operated on with intraoperative imaging guidance $\left(\mathrm{O}-\mathrm{arm}^{\odot}\right)$ and outcomes were in the same range as those provided in the literature.

Finally, Chen et al. [21] published data of a single-center prospective nonrandomized study. Asleep or awake surgery was performed according to the patient's own preference and approval by the movement disorder neurologist. This trial had the advantage of homogenizing the surgical techniques (including target planning and contemporary stereotactic procedure), outcome measures, and follow-up between the 2 groups because of its singlecenter characteristic but was subject to selection bias because of the nonrandomized design. In this study, 41 pa-

Asleep versus Awake Subthalamic

Nucleus Deep Brain Stimulation tients underwent asleep DBS surgery and 14 underwent awake DBS surgery. The primary outcome measure was the stimulation-induced change in motor function 6 months after surgery, as in our study. The percentage change was similar between awake (40.3\%) and asleep $(48.8 \%)$ groups $(p=0.06)$. Concerning adverse events, no patients had hemorrhage or infection, and with the exception of 2 cases of hypomania in the awake group $(p=$ 0.001 ), intergroup differences in the frequency of specific complications did not differ significantly.

Recent advances in neuroimaging have changed the paradigm of STN-DBS targeting with improved visualization of the STN. On the one hand, T2 weighted imaging sequence has been shown to correlate well with the neurophysiological target in most of the cases [37], and more recently quantitative susceptibility mapping has shown promising images in direct targeting of the STN [28]. However, on the other hand, some authors argue that MER may be still useful to improve implantation accuracy $[30,38]$. The potential interest of direct imagebased STN targeting has only been studied by comparing the positions of the planned target (image-based) with the MER-guided adjusted target and the final lead during standard awake procedures [29, 30, 37, 39-41]. However, to our knowledge, there is no study that specifically compared the clinical outcomes after implantation using imaging alone (without MER and clinical testing) versus the same image-based targeting corrected by MER and clinical testing. The same remarks apply to deformable atlasbased targeting [27, 42-45].

The paradigm of DBS has also evolved with the high level of lead placement accuracy obtained by modern stereotactic techniques. Indeed, the role of intraoperative MER has always been subject to debate and 18 years ago we already raised the question whether MER adjusted a suboptimal intended lead placement (based on stereotactic coordinates and/or imaging) or corrected a trajectory that has deviated from the planned target during the procedure [40]. This debate is illustrated in our study by the fact that although the small sample size precludes any definitive conclusion, we did not find larger intended errors in the awake group. The primordial role of stereotactic accuracy on surgical outcomes has been well illustrated by Chen et al. $[46,47]$ with patients who underwent asleep Vim-DBS for essential tremor. Targeting was performed upon the same set of standardized stereotactic coordinates $(x=10.5 \mathrm{~mm}$ lateral to the third ventricle; $y=25 \%$ of the AC-PC distance posterior to the midcommissural point; and $z=0$ ) without any atlas or image-guided planification. The authors showed that the percentage of tremor im- 
provement at an average follow-up of 17 months was not significantly different from the improvement after standard awake DBS with MER and intraoperative test stimulation. None of the 17 asleep patients required returning to the operating room within 6 months for lead repositioning due to adverse stimulation effects. Furthermore, the final Euclidean error of the DBS lead was significantly higher in the awake group (1.7 vs. $1.2 \mathrm{~mm}, p=0.01$ ).

In our center, since 2012, we have taken the lead to develop an original targeting method different from direct image-based or indirect atlas-based or standard stereotactic coordinate-based targeting. Indeed, outcomes after STN-DBS are not well predicted by the classical clinical criteria required for a DBS procedure for PD patients [48], nor by the DBS-lead positioning in the so-called subthalamus nucleus target whose exact nature remains controversial (the STN [10] itself, the border of the STN [49-51], and the zona incerta and posterior subthalamic area [5257]). These difficulties could be explained by the limits of the methods used to select the patients for STN-DBS (clinical criteria) and to control DBS-lead anatomical location on postoperative imaging $[58,59]$. To try to overcome these difficulties, we described a clinical-based STN target based on the resolution of what is called in mathematics an inverse problem (for further details on the definition of the target, see reference [33]). Our clinical STN target is related to 3 landmarks well identifiable on a T1 MRI (the mammillothalamic tract $4 \mathrm{~mm}$ below the AC-PC line, the lateral wall of the third ventricle at the midcommissural point, and the maximum height of the thalamus on sagittal slices) which were significantly correlated to the DBSlead locations in a cohort of patients with optimal motor outcomes after STN-DBS. Furthermore, this target takes advantage of an easy identification of the landmarks with high reproducibility on a T1 MRI at $1.5 \mathrm{~T}$ or $3 \mathrm{~T}$ which can be used in routine DBS procedures without the need of image coregistration, normalization, or any other postprocessing procedure which may add uncontrollable errors in the target identification process and thus decrease the global accuracy of the DBS procedure.

In addition to this "clinical" targeting, a high level of lead placement accuracy was achieved in our study by the use of a surgical robot (Neuromate ${ }^{\circledR}$; Renishaw Inc.) under intraoperative imaging guidance $\left(\mathrm{O}-\operatorname{arm}^{\odot}\right.$; Medtronic Inc.). Furthermore, in the asleep group, GA may have optimized the lead placement by taking advantage of stricter blood pressure control, less cerebrospinal fluid loss, and decreased patient and brain movements.

The main limitation of this study is the small sample size that did not allow for formal statistical testing of the hypothesis of the noninferiority of the asleep procedure with regard to the awake procedure. However, our study yielded valid between-group difference estimates that will be used for sample size calculation for a phase 3 multicenter controlled randomized noninferiority trial (PARKEO 2 trial) which should start shortly in France.

\section{Conclusion}

Owing to its randomized design, our study supports the hypothesis that motor outcomes after asleep STNDBS in PD may be equivalent to the standard awake procedure with MER and clinical testing.

\section{Acknowledgements}

The authors thank Marie Eresue-Bony and Stéphanie Mora (radiophysics engineer) for providing data on irradiation, MarieClaire Toussaint (coordinating nurse of the movement disorders center of our institution) for helping with the collection of data on quality of life, and Jérôme Galet (biostatistician) for data management of this trial.

\section{Statement of Ethics}

The trial protocol was approved by the regional scientific ethics committee (Comité de Protection des Personnes Sud-Ouest et Outre-Mer III) and was performed in accordance with the principles of the Declaration of Helsinki. This study is registered with ClinicalTrials.gov, No. NCT01817088. Written informed consent was obtained from all patients.

\section{Conflict of Interest Statement}

Prof. Emmanuel Cuny received consultant fees from Medtronic, which manufactures the devices used in this trial. Other authors report no conflicts of interest.

\section{Funding Sources}

This trial was supported by a grant of the Bordeaux University Hospital and grant from the Medtronic ${ }^{\mathrm{TM}}$ society.

\section{Author Contributions}

D.G., O.B., A.B., R.S., F.C., X.A., P.B., and E.C.: conception and design of the study. J.E., N.D.P., D.G., O.B., N.A., F.T., W.M., E.K., S.B., A.B., R.S., D.F., P.B., and E.C.: acquisition and analysis of the data. J.E., A.B., F.C., P.B., and E.C.: drafting a significant portion of the manuscript. 


\section{References}

1 Anderson VC, Burchiel KJ, Hogarth P, Favre J, Hammerstad JP. Pallidal vs subthalamic nucleus deep brain stimulation in Parkinson disease. Arch Neurol. 2005 Apr;62(4):554-60.

2 Deuschl G, Schade-Brittinger C, Krack P, Volkmann J, Schäfer H, Bötzel K, et al. A randomized trial of deep-brain stimulation for Parkinson's disease. N Engl J Med. 2006; 355(9):896.

3 Kleiner-Fisman G, Herzog J, Fisman DN, Tamma F, Lyons KE, Pahwa R, et al. Subthalamic nucleus deep brain stimulation: summary and meta-analysis of outcomes. Mov Disord. 2006 Jun;21(Suppl 14):S290-304.

4 Odekerken VJ, Boel JA, Schmand BA, de Haan RJ, Figee M, van den Munckhof P, et al. GPi vs STN deep brain stimulation for Parkinson disease: three-year follow-up. Neurology. 2016 Feb;86(8):755-61.

5 Okun MS, Gallo BV, Mandybur G, Jagid J, Foote KD, Revilla FJ, et al. Subthalamic deep brain stimulation with a constant-current device in Parkinson's disease: an open-label randomised controlled trial. Lancet Neurol. 2012 Feb;11(2):140-9.

6 Schüpbach WM, Rau J, Knudsen K, Volkmann J, Krack P, Timmermann L, et al. Neurostimulation for Parkinson's disease with early motor complications. N Engl J Med. 2013 Feb;368(7):610-22.

7 Schüpbach WMM, Maltete D, Houeto JL, du Montcel ST, Mallet L, Welter ML, et al. Neurosurgery at an earlier stage of Parkinson disease: a randomized, controlled trial. Neurology. 2007 Jan;68(4):267-71.

8 Weaver FM, Follett K, Stern M, Hur K, Harris C, Marks WJ, et al. Bilateral deep brain stimulation vs best medical therapy for patients with advanced Parkinson disease: a randomized controlled trial. JAMA. 2009 Jan;301(1): 63.

9 Williams A, Gill S, Varma T, Jenkinson C, Quinn N, Mitchell R, et al. Deep brain stimulation plus best medical therapy versus best medical therapy alone for advanced Parkinson's disease (PD SURG trial): a randomised, open-label trial. Lancet Neurol. 2010 Jun;9(6): 581-91.

10 Welter ML, Schüpbach M, Czernecki V, Karachi C, Fernandez-Vidal S, Golmard JL, et al. Optimal target localization for subthalamic stimulation in patients with Parkinson disease. Neurology. 2014 Apr;82(15):1352-61.

11 Limousin P, Krack P, Pollak P, Benazzouz A, Ardouin C, Hoffmann D, et al. Electrical stimulation of the subthalamic nucleus in advanced Parkinson's disease. N Engl J Med. 1998 Oct;339(16):1105-11.

12 Zrinzo L, Foltynie T, Limousin P, Hariz MI. Reducing hemorrhagic complications in functional neurosurgery: a large case series and systematic literature review. J Neurosurg. 2012 Jan;116(1):84-94.
13 Jacob RL, Geddes J, McCartney S, Burchiel KJ. Cost analysis of awake versus asleep deep brain stimulation: a single academic health center experience. J Neurosurg. 2016 May; 124(5):1517-23.

14 Brontë-Stewart H, Louie S, Batya S, Henderson JM. Clinical motor outcome of bilateral subthalamic nucleus deep-brain stimulation for Parkinson's disease using image-guided frameless stereotaxy. Neurosurgery. 2010 Oct; 67(4):1088-93.

15 Burchiel KJ, McCartney S, Lee A, Raslan AM. Accuracy of deep brain stimulation electrode placement using intraoperative computed tomography without microelectrode recording. J Neurosurg. 2013 Aug;119(2):301-6.

16 Harries AM, Kausar J, Roberts SA, Mocroft AP, Hodson JA, Pall HS, et al. Deep brain stimulation of the subthalamic nucleus for advanced Parkinson disease using general anesthesia: long-term results. J Neurosurg. 2012 Jan;116(1):107-13.

17 Larson PS, Starr PA, Bates G, Tansey L, Richardson RM, Martin AJ. An optimized system for interventional magnetic resonance imaging-guided stereotactic surgery: preliminary evaluation of targeting accuracy. Neurosurgery. 2012 Mar;70(1 Suppl Operative):95103.

18 Maltête D, Navarro S, Welter ML, Roche S, Bonnet AM, Houeto JL, et al. Subthalamic stimulation in Parkinson disease: with or without anesthesia? Arch Neurol. 2004 Mar; 61(3):390

19 Blasberg F, Wojtecki L, Elben S, Slotty PJ, Vesper J, Schnitzler A, et al. Comparison of awake vs. asleep surgery for subthalamic deep brain stimulation in Parkinson's disease: awake vs. asleep STN-DBS surgery in PD. Neuromodulation. 2018 Aug;21(6):541-7.

20 Brodsky MA, Anderson S, Murchison C, Seier M, Wilhelm J, Vederman A, et al. Clinical outcomes of asleep vs awake deep brain stimulation for Parkinson disease. Neurology. 2017 Nov;89(19): 1944-50.

21 Chen T, Mirzadeh Z, Chapple KM, Lambert M, Shill HA, Moguel-Cobos G, et al. Clinical outcomes following awake and asleep deep brain stimulation for Parkinson disease. J Neurosurg. 2018 Mar;130(1):109-20.

22 Chen T, Mirzadeh Z, Ponce FA. "Asleep" deep brain stimulation surgery: a critical review of the literature. World Neurosurg. 2017 Sep; $105: 191-8$.

23 Ho AL, Ali R, Connolly ID, Henderson JM, Dhall R, Stein SC, et al. Awake versus asleep deep brain stimulation for Parkinson's disease: a critical comparison and meta-analysis. J Neurol Neurosurg Psychiatry. 2018 Jul; 89(7):687-91.

24 Liu Z, He S, Li L. General anesthesia versus local anesthesia for deep brain stimulation in Parkinson's disease: a meta-analysis. Stereotact Funct Neurosurg. 2019;97(5-6):381-90.
25 Saleh S, Swanson KI, Lake WB, Sillay KA. Awake neurophysiologically guided versus asleep MRI-guided STN DBS for Parkinson disease: a comparison of outcomes using levodopa equivalents. Stereotact Funct Neurosurg. 2015;93(6):419-26.

26 Sheshadri V, Rowland NC, Mehta J, Englesakis M, Manninen P, Venkatraghavan L. Comparison of general and local anesthesia for deep brain stimulator insertion: a systematic review. Can J Neurol Sci. 2017 Nov;44(6):1-8.

27 Ewert S, Plettig P, Li N, Chakravarty MM, Collins DL, Herrington TM, et al. Toward defining deep brain stimulation targets in MNI space: a subcortical atlas based on multimodal MRI, histology and structural connectivity. NeuroImage. 2018 Apr;170:271-82.

28 Rasouli J, Ramdhani R, Panov FE, Dimov A, Zhang Y, Cho C, et al. Utilization of quantitative susceptibility mapping for direct targeting of the subthalamic nucleus during deep brain stimulation surgery. Oper Neurosurg. 2018 Apr;14(4):412-9.

29 Shamir RR, Duchin Y, Kim J, Patriat R, Marmor O, Bergman $\mathrm{H}$, et al. Microelectrode recordings validate the clinical visualization of subthalamic-nucleus based on $7 \mathrm{~T}$ magnetic resonance imaging and machine learning for deep brain stimulation surgery. Neurosurgery. 2019 Mar;84(3):749-57.

30 Lozano CS, Ranjan M, Boutet A, Xu DS, Kucharczyk W, Fasano A, et al. Imaging alone versus microelectrode recording-guided targeting of the STN in patients with Parkinson's disease. J Neurosurg. 2018 [Epub ahead of print].

31 Grinsztejn B, De Castro N, Arnold V, Veloso VG, Morgado M, Pilotto JH, et al. Raltegravir for the treatment of patients co-infected with HIV and tuberculosis (ANRS 12180 Reflate $\mathrm{TB}$ ): a multicentre, phase 2, non-comparative, open-label, randomised trial. Lancet Infect Dis. 2014 Jun;14(6):459-67.

32 Richert L, Doussau A, Lelièvre JD, Arnold V, Rieux V, Bouakane A, et al. Accelerating clinical development of HIV vaccine strategies: methodological challenges and considerations in constructing an optimised multiarm phase I/II trial design. Trials. $2014 \mathrm{Feb}$; 15:68.

33 Caire F. Imagerie per-opératoire des électrodes de stimulation cérébrale profonde et proposition d'une nouvelle modalité de repérage stéréotaxique indirect de la cible subthalamique. $2012 \mathrm{Dec}$. Available from: https:// www.theses.fr/082391483.

34 Caire F, Maubon A, Moreau JJ, Cuny E. The mamillothalamic tract is a good landmark for the anterior border of the subthalamic nucleus on axial MR images. Stereotact Funct Neurosurg. 2011;89(5):286-90.

35 Harrington D, D'Agostino RB, Gatsonis C, Hogan JW, Hunter DJ, Normand ST, et al. New guidelines for statistical reporting in the Journal. N Engl J Med. 2019 Jul;381(3):285-6.
Asleep versus Awake Subthalamic

Nucleus Deep Brain Stimulation
Stereotact Funct Neurosurg 2021;99:230-240 DOI: $10.1159 / 000511424$ 
36 Kochanski R, Sani S. Awake versus asleep deep brain stimulation surgery: technical considerations and critical review of the literature. Brain Sci. 2018 Jan;8(1):17.

37 Nowacki A, Debove I, Fiechter M, Rossi F, Oertel MF, Wiest R, et al. Targeting accuracy of the subthalamic nucleus in deep brain stimulation surgery: comparison between $3 \mathrm{~T} \mathrm{~T} 2$ weighted magnetic resonance imaging and microelectrode recording results. Oper Neurosurg. 2018 Jul;15(1):66-71.

38 Ranjan M, Boutet A, Xu DS, Lozano CS, Kumar R, Fasano A, et al. Subthalamic nucleus visualization on routine clinical preoperative MRI scans: a retrospective study of clinical and image characteristics predicting its visualization. Stereotact Funct Neurosurg. 2018; 96(2):120-6.

39 Chandran AS, Bynevelt M, Lind CR. Magnetic resonance imaging of the subthalamic nucleus for deep brain stimulation. J Neurosurg. 2016 Jan;124(1):96-105.

40 Cuny E, Guehl D, Burbaud P, Gross C, Dousset $V$, Rougier A. Lack of agreement between direct magnetic resonance imaging and statistical determination of a subthalamic target: the role of electrophysiological guidance. J Neurosurg. 2002 Sep;97(3):591-7.

41 Polanski WH, Martin KD, Engellandt K, von Kummer R, Klingelhoefer L, Fauser M, et al. Accuracy of subthalamic nucleus targeting by T2, FLAIR and SWI-3-Tesla MRI confirmed by microelectrode recordings. Acta Neurochir. 2015 Mar;157(3):479-86.

42 Bardinet E, Bhattacharjee M, Dormont D, Pidoux B, Malandain G, Schüpbach M, et al. A three-dimensional histological atlas of the human basal ganglia. II. Atlas deformation strategy and evaluation in deep brain stimulation for Parkinson disease. J Neurosurg. 2009 Feb;110(2):208-19.

43 Horn A, Li N, Dembek TA, Kappel A, Boulay C, Ewert S, et al. Lead-DBS v2: towards a comprehensive pipeline for deep brain stimulation imaging. Neuroimage. 2019 Jan 1;184 293-316.
44 Welter ML, Schüpbach M, Czernecki V, Karachi C, Fernandez-Vidal S, Golmard JL, et al. Optimal target localization for subthalamic stimulation in patients with Parkinson disease. Neurology. 2014 Apr;82(15):1352-61.

45 Yelnik J, Bardinet E, Dormont D, Malandain G, Ourselin S, Tandé D, et al. A three-dimensional, histological and deformable atlas of the human basal ganglia. I. Atlas construction based on immunohistochemical and MRI data. NeuroImage. 2007 Jan;34(2):618-38.

46 Chen T, Mirzadeh Z, Chapple K, Lambert M, Dhall R, Ponce FA. "Asleep” deep brain stimulation for essential tremor. J Neurosurg. 2016 Jun;124(6):1842-9.

47 Chen T, Mirzadeh Z, Chapple KM, Lambert M, Evidente VGH, Moguel-Cobos G, et al. Intraoperative test stimulation versus stereotactic accuracy as a surgical end point: a comparison of essential tremor outcomes after ventral intermediate nucleus deep brain stimulation. J Neurosurg. 2018 Aug; 129(2): 290-8.

48 Hartmann CJ, Fliegen S, Groiss SJ, Wojtecki L, Schnitzler A. An update on best practice of deep brain stimulation in Parkinson's disease. Ther Adv Neurol Disord. 2019; 12: 1756286419838096.

49 Herzog J, Fietzek U, Hamel W, Morsnowski A, Steigerwald F, Schrader B, et al. Most effective stimulation site in subthalamic deep brain stimulation for Parkinson's disease. Mov Disord. 2004 Sep;19(9):1050-4.

50 Johnsen EL, Sunde N, Mogensen PH, Ostergaard K. MRI verified STN stimulation site: gait improvement and clinical outcome. Eur J Neurol. 2010 May;17(5):746-53.

51 Zonenshayn M, Sterio D, Kelly PJ, Rezai AR, Beric A. Location of the active contact within the subthalamic nucleus (STN) in the treatment of idiopathic Parkinson's disease. Surg Neurol. 2004 Sep;62(3):216-25; discussion 225-6.

52 Guehl D, Vital A, Cuny E, Spampinato U, Rougier A, Bioulac B, et al. Postmortem proof of effectiveness of zona incerta stimulation in Parkinson disease. Neurology. 2008 Apr; 70(16 Pt 2):1489-90.
53 Hamel W, Fietzek U, Morsnowski A, Schrader B, Herzog J, Weinert D, et al. Deep brain stimulation of the subthalamic nucleus in Parkinson's disease: evaluation of active electrode contacts. J Neurol Neurosurg Psychiatry. 2003 Aug;74(8):1036-46.

54 Maks CB, Butson CR, Walter BL, Vitek JL, McIntyre CC. Deep brain stimulation activation volumes and their association with neurophysiological mapping and therapeutic outcomes. J Neurol Neurosurg Psychiatry. 2009 Jun;80(6):659-66.

55 Plaha P, Ben-Shlomo Y, Patel NK, Gill SS. Stimulation of the caudal zona incerta is superior to stimulation of the subthalamic nucleus in improving contralateral parkinsonism. Brain. 2006 Jul;129(Pt 7):1732-47.

56 Vergani F, Landi A, Antonini A, Parolin M, Cilia R, Grimaldi M, et al. Anatomical identification of active contacts in subthalamic deep brain stimulation. Surg Neurol. 2007 Feb; 67(2):140-7; discussion 146-7.

57 Voges J, Volkmann J, Allert N, Lehrke R, Koulousakis A, Freund HJ, et al. Bilateral high-frequency stimulation in the subthalamic nucleus for the treatment of Parkinson disease: correlation of therapeutic effect with anatomical electrode position. J Neurosurg. 2002 Feb;96(2):269-79.

58 Engelhardt J, Guehl D, Damon-Perrière N, Branchard O, Burbaud P, Cuny E. Localization of deep brain stimulation electrode by image registration is software dependent: a comparative study between four widely used software programs. Stereotact Funct Neurosurg. 2018;96(6):364-9.

59 Verhagen R, Schuurman PR, van den Munckhof P, Contarino MF, de Bie RM, Bour LJ. Comparative study of microelectrode recording-based STN location and MRI-based STN location in low to ultra-high field (7.0 T) T2weighted MRI images. J Neural Eng. 2016; 13(6):066009. 